\title{
Semen Quality and Sperm DNA Damage in Relation to Urinary Bisphenol A among Men from an Infertility Clinic
}

\author{
John D. Meeker ${ }^{1}$, Shelley Ehrlich ${ }^{2}$, Thomas L. Toth ${ }^{3}$, Diane L. Wright ${ }^{3}$, Antonia M. \\ Calafat $^{4}$, Ana T. Trisini ${ }^{2}$, Xiaoyun $\mathrm{Ye}^{4}$, and Russ Hauser ${ }^{2,3}$ \\ ${ }^{1}$ Department of Environmental Health Sciences, University of Michigan, Ann Arbor, MI \\ ${ }^{2}$ Department of Environmental Health, Harvard School of Public Health, Boston, MA \\ ${ }^{3}$ Vincent Memorial Obstetrics and Gynecology Service, Massachusetts General Hospital, Boston, \\ MA \\ ${ }^{4}$ Centers for Disease and Control and Prevention, Atlanta, GA
}

\begin{abstract}
Bisphenol A (BPA) impairs spermatogenesis in animals, but human studies are lacking. We measured urinary BPA concentrations, semen quality, and sperm DNA damage (comet assay) in 190 men recruited through an infertility clinic. BPA was detected in $89 \%$ of samples, with a median (interquartile range [IQR]) concentration of $1.3(0.8-2.5) \mathrm{ng} / \mathrm{mL}$. Urinary BPA concentration was associated with slightly elevated, though not statistically significant, odds for below reference sperm concentration, motility, and morphology. When modeled as continuous dependent variables, an IQR increase in urinary BPA concentration was associated with declines in sperm concentration, motility, and morphology of $23 \%(95 \% \mathrm{CI}-40 \%,-0.3 \%), 7.5 \%(-17 \%$, $+1.5 \%)$, and $13 \%(-26 \%,-0.1 \%)$, respectively, along with a $10 \%(0.03 \%, 19 \%)$ increase in sperm DNA damage measured as the percentage of DNA in comet tail. In conclusion, urinary BPA may be associated with declined semen quality and increased sperm DNA damage, but confirmatory studies are needed.
\end{abstract}

\section{Keywords}

Biomarkers; Endocrine Disruption; Environment; Epidemiology; Estrogenic; Exposure; Fertility; Male; Reproduction

\section{INTRODUCTION}

Bisphenol A (BPA) is a high production volume chemical used in the manufacture of polycarbonate plastics, which can be used in baby and water bottles, and epoxy resins, used in food container linings and other applications; the use of these products can lead to human

(C) 2010 Elsevier Inc. All rights reserved.

Address correspondence to: John Meeker, ScD Department of Environmental Health Sciences University of Michigan School of Public Health 6635 SPH Tower 109 S. Observatory St. Ann Arbor, MI 48109 Phone: 1-734-764-7184 Fax: 1-734-936-7283 meekerj@umich.edu.

Publisher's Disclaimer: This is a PDF file of an unedited manuscript that has been accepted for publication. As a service to our customers we are providing this early version of the manuscript. The manuscript will undergo copyediting, typesetting, and review of the resulting proof before it is published in its final citable form. Please note that during the production process errors may be discovered which could affect the content, and all legal disclaimers that apply to the journal pertain.

Conflict of Interest Statement: The authors declare that there are no conflicts of interest. 
exposure [1]. Over 6 billion pounds of BPA are produced annually, with a 6-10\% growth in demand expected per year [2]. Dietary ingestion is considered the primary source of general population exposure to BPA. Other exposure sources may include water, air, and dust $[1,3]$. As a result, there is widespread general population exposure to BPA $[1,4]$. BPA has been shown to alter endocrine function through multiple pathways [5], and a number of animal studies have reported adverse reproductive effects in males exposed to low levels of BPA in early life or in adulthood [6]. To our knowledge no human studies of BPA exposure and semen quality or sperm DNA damage have been conducted to date. In the present study we assessed the relationship between urinary BPA concentrations, which have been used as a biomarker of exposure to BPA [4], and semen quality and sperm DNA damage in men recruited through a United States (US) infertility clinic.

\section{METHODS}

Subjects were recruited during 2000-2004 from an ongoing study on the relationship between environmental agents and reproductive health. Participating men were partners in subfertile couples seeking treatment from the Vincent Andrology lab at Massachusetts General Hospital. The study was approved by the Human Studies Institutional Review Boards of the Massachusetts General Hospital, Harvard School of Public Health, the Centers for Disease Control and Prevention (CDC), and the University of Michigan. After the study procedures were explained and all questions answered, subjects signed an informed consent. Men between the ages of 18 to 55 years without post-vasectomy status who presented to the Andrology Laboratory were eligible to participate. Of those approached, approximately $65 \%$ consented. Most men that declined to participate in the study cited lack of time on the day of their clinic visit as the reason for not participating.

\section{Urinary BPA}

A single spot urine sample was collected from each subject on the day of their clinic visit in a sterile polypropylene cup. Because BPA is metabolized and excreted from the body rapidly, and a single urinary measure likely reflects an individual's exposure in the hours to days leading up to urine sample collection [7], a second and third urine sample were collected from a subset of men. These samples were generally collected between one week and two months following the first sample at a follow-up clinic visit. After measuring specific gravity (SG) using a handheld refractometer (National Instrument Company, Inc., Baltimore, MD, USA), each urine sample was divided in aliquots and frozen at $-80^{\circ} \mathrm{C}$. Samples were shipped on dry ice overnight to the CDC, Atlanta, Georgia, USA, where the total urinary concentration of BPA (free plus conjugated species) was measured using online solid-phase extraction (SPE) coupled to isotope dilution-high performance liquid chromatography (HPLC) - tandem mass spectrometry (MS/MS) on a system constructed from several HPLC Agilent 1100 modules (Agilent Technologies, Wilmington, DE) coupled to a triple quadropole API 4000 mass spectrometer (Applied Biosystems, Foster City, CA) [8]. First, $100 \mu \mathrm{L}$ of urine was treated with $\beta$-glucuronidase/sulfatase (Helix pomatia, H1; Sigma Chemical Co., St. Louis, MO) to hydrolyze the BPA-conjugated species. BPA was then retained and concentrated on a C18 reversed-phase size-exclusion SPE column (Merck $\mathrm{KGaA}$, Germany), separated from other urine matrix components using a pair of monolithic HPLC columns (Merck KGaA), and detected by negative ion-atmospheric pressure chemical ionization-MS/MS. The limit of detection (LOD) for BPA in a 0.1-mL urine sample was 0.4 $\mathrm{ng} / \mathrm{mL}$. Low-concentration $(\sim 4 \mathrm{ng} / \mathrm{mL})$ and high-concentration $(\sim 20 \mathrm{ng} / \mathrm{mL})$ quality control materials, prepared with pooled human urine, were analyzed with analytical standards, reagent blanks, and unknown samples [8]. Analysts at the CDC were blind to all information concerning subjects. BPA concentrations were corrected for urine dilution by specific gravity (SG) using the following formula: $\left.\mathrm{P}_{\mathrm{c}}=\mathrm{P}[(1.024-1) / \mathrm{SG}-1)\right]$, where $\mathrm{P}_{\mathrm{c}}$ is the SG- 
adjusted urinary BPA concentration $(\mathrm{ng} / \mathrm{mL}), \mathrm{P}$ is the observed BPA concentration, and SG is the specific gravity of the urine sample. SG was measured using a handheld refractometer (National Instrument Company, Inc., Baltimore, MD, USA).

\section{Semen sample collection}

Semen was collected on site at Massachusetts General Hospital in a sterile plastic specimen cup after a recommended period of abstinence of 48 hours. After liquefaction at $37^{\circ} \mathrm{C}$ for 30 minutes, semen quality parameters and motion characteristics were measured at the clinic. The remaining unprocessed semen was frozen in $0.25 \mathrm{~mL}$ cryogenic straws (CryoBiosystem, I.M.V. Division, San Diego, CA) by immersing the straws directly into liquid nitrogen (-196 $\left.{ }^{\circ} \mathrm{C}\right)$. Previous work in our laboratory showed that this freezing method produced comet assay results that were highly correlated with results from fresh, unfrozen samples [9]. Semen samples were later analyzed in batches, where straws were thawed by gently shaking in a $37{ }^{\circ} \mathrm{C}$ water bath for 10 seconds and the semen was immediately processed for the comet assay.

\section{Semen quality}

In the MGH Andrology Laboratory, trained clinical staff analyzed semen samples for sperm concentration, total sperm count, and motion parameters using computer-aided semen analyzer (CASA, HTM-IVOS Version 10HTM-IVOS, Beverly, MA, USA). Setting parameters and the definition of measured sperm motion parameters for CASA were established by the Hamilton-Thorn Company. To measure both sperm concentration and motility, $5 \mu \mathrm{L}$ of semen from each sample was placed into a pre-warmed $\left(37^{\circ} \mathrm{C}\right)$ Makler counting chamber (Sefi - Medical instruments, Haifa, Israel). A minimum of 200 sperm cells from at least four different fields was analyzed from each specimen. Total sperm count $\left(10^{6} /\right.$ ejaculate) was calculated by multiplying sperm concentration $\left(10^{6} / \mathrm{mL}\right)$ by semen sample volume $(\mathrm{mL})$. Motile sperm was defined as World Health Organization (WHO) grade "a" sperm (rapidly progressive with a velocity $\geq 25 \mu \mathrm{m} / \mathrm{s}$ at $37^{\circ} \mathrm{C}$ ) plus "b" grade sperm (slow/sluggish progressive with a velocity $\geq 5 \mu \mathrm{m} / \mathrm{s}$ but $<25 \mu \mathrm{m} / \mathrm{s}$ ) [10]. Measurement of CASA motion characteristics has been previously described [11,12]. Of seven CASA variables that were measured, only three were chosen (straight-line velocity (VSL), curvilinear velocity $(\mathrm{VCL})$ and linearity $(\mathrm{LIN}=\mathrm{VSL} / \mathrm{VCL} \times 100)$ ) for inclusion in the present analysis due to a high degree of dependence between several of the measures.

At least two slides were made for each fresh semen sample. The resulting thin smear was allowed to air dry for an hour before staining with a Diff-Quik staining kit (Dade Behring AG, Dudingen, Switzerland). Morphological assessment was performed with a Nikon microscope using an oil immersion 100X objective (Nikon Company, Tokyo, Japan). A minimum of 200 sperm cells was counted from two slides for each specimen. Strict Kruger scoring criteria were used to classify men as having normal or below normal morphology [13].

\section{Neutral Comet assay}

The comet assay procedure used in the present study to assess sperm DNA damage has been previously described [14,15]. Briefly, $50 \mu \mathrm{L}$ of a semen/agarose mixture $(0.7 \% 3: 1$ high resolution agarose; Amresco, Solon, OH, USA) was embedded between two additional layers of agarose on microgel electrophoresis glass slides (Erie Scientific, Portsmouth, NH, USA). Slides were then immersed in a cold lysing solution to dissolve the cell membrane and make chromatin accessible for the enzyme digestion steps. After 1 hour cold lysis, slides were transferred to a solution for enzyme treatment with $10 \mu \mathrm{g} / \mathrm{mL}$ of RNase (Amresco, Solon, OH, USA) and incubated at $37^{\circ} \mathrm{C}$ for 4 hours. Slides were then transferred to a second enzyme treatment with $1 \mathrm{mg} / \mathrm{mL}$ proteinase K (Amresco, Solon, OH, USA) and 
incubated at $37^{\circ} \mathrm{C}$ for 18 hours. The slides were placed on a horizontal slab in an electrophoretic unit, equilibrated for 20 minutes, and underwent electrophoresis for 1 hour. DNA in the gel was then precipitated, fixed in ethanol, and dried. Slides were stained and observed with fluorescence microscope. Comet extent, tail distributed moment (TDM), and percent DNA located in the tail (Tail\%) were measured on 100 sperm in each semen sample using VisComet software (Impuls Computergestutzte Bildanalyse $\mathrm{GmbH}$, Gilching, Germany). Comet extent is a measure of total comet length from the beginning of the head to the last visible pixel in the tail. Tail\% is a measurement of the proportion of total DNA that is present in the tail. TDM is an integrated value that takes into account both the distance and intensity of comet fragments:

$$
\mathrm{TDM}=\Sigma\left(\mathrm{I}^{*} \mathrm{X}\right) / \Sigma \mathrm{I}
$$

where $\Sigma \mathrm{I}$ is the sum of all intensity values that belong to the head, body, or tail, and $\mathrm{X}$ is the $\mathrm{x}$-position of the intensity value.

\section{Statistical analysis}

Data analysis was performed using SAS version 9.1 (SAS Institute Inc., Cary, NC, USA). Descriptive statistics on subject demographics were calculated, along with the distributions of urinary BPA concentrations, semen quality, and sperm DNA damage measures. To investigate differences between distributions or categories and the potential for confounding, bivariate analysis was conducted between urinary BPA concentrations, and semen quality, sperm DNA damage, and demographic variables. Differences were tested statistically using parametric or non-parametric methods where appropriate. We also downloaded the most recent publicly available urinary BPA data reported by the US National Health and Nutrition Examination Survey (NHANES 2005-06) to compare BPA concentrations in our study with those measured among men in the same age range in the general population [16].

The association between urinary BPA concentrations and sperm concentration, motility and morphology were first assessed by multiple logistic regression, where subjects were dichotomized as either below or equal/above WHO reference levels for total sperm count (40 million sperm), sperm concentration (20 million sperm $/ \mathrm{mL}$ ) and sperm motility (50\% motile sperm) [10]. The strict Kruger criteria (4\% normal) was used as a cutoff for sperm morphology [13]. Comparison subjects were those that were above the reference level for all three parameters. Urinary BPA concentrations were lognormally distributed and transformed by the natural logarithm (ln) prior to statistical analysis. Samples with BPA concentrations below the LOD were assigned a value equal to $1 / 2$ the LOD in the statistical analyses. In all models, specific gravity was included as a continuous variable to adjust for urinary dilution. Age, body mass index (BMI), race, abstinence period, smoking, and time of day of the clinic visit (time urine/semen samples were collected: morning [9:00 am - 12:59 pm] vs. afternoon [1:00 pm - 4:00 pm]) were considered as covariates, and were included or excluded from models based on biologic and statistical considerations [17]. Covariates were included in the model if they are known to be biologically important (i.e. abstinence period) or they acted as a confounder (i.e. changed the BPA parameter estimate by $>10 \%$ ) in any of the statistical models; all models were adjusted for the same covariates for consistency. To improve interpretability, all models were adjusted for the same covariates and effect estimates were expressed as odds ratios (OR) associated with an interquartile range (IQR) increase in urinary BPA concentrations.

Multiple linear regression was also used to assess associations between urinary BPA concentrations and continuous measures of semen quality, sperm motion parameters, and sperm DNA damage. Total sperm count and sperm concentration were transformed using 
the natural logarithm, whereas all other semen quality, sperm motion, and DNA damage measures were modeled untransformed. Urinary BPA concentrations were also lntransformed. The same covariates listed above for the logistic regression models were considered, and specific gravity was again included as a continuous variable in all models to adjust for urinary dilution. To improve interpretability, the regression coefficients were back transformed and expressed as a change in the dependent variable (i.e., semen quality or sperm DNA damage measures) for an interquartile range (IQR) increase in urinary BPA concentrations.

Four sets of models were constructed: 1) using only urinary BPA concentrations from a single urine sample collected on the same day as the semen sample; 2) using the geometric mean urinary BPA concentration for each participant, where between one and three values were used to calculate each individual's geometric mean (i.e. the geometric mean for men with only one urine sample was equal to that single value); 3) using the geometric mean urinary BPA concentration among only participants that contributed BPA data from at least two urine samples; and 4) using only the single urinary BPA measure collected on the same day as the semen sample among men with BPA data from at least two urine samples. The reason for including approach 4) was to assess the utility of collecting subsequent urine samples by comparing effect estimates between approaches 3 ) and 4). In a sensitivity analysis, the multivariable models were rerun after excluding men with highly concentrated or highly dilute urine samples (SG above 1.03 or below 1.01) [18]. Models were also rerun using specific gravity-corrected BPA concentrations rather than using uncorrected urinary BPA concentrations but including specific gravity as a covariate. In addition, we assessed non-linear relationships of urinary BPA concentrations with semen quality and sperm DNA damage parameters by categorizing urinary BPA concentrations into quartiles in both linear and logistic regression models. Finally, we modeled relationships between urinary BPA and semen quality parameters in a stratified analysis where effect estimates from men with at least one semen parameter below WHO reference values were compared to those among men with all semen parameters above WHO reference.

\section{RESULTS}

Urinary BPA concentrations, specific gravity, and semen quality parameters from samples collected at the same clinic visit were available for 190 men. Of these men, a second urine sample was later collected from 78 of them, and a third urine sample was collected from 4 men. As a result, the total number of urine samples collected and analyzed for BPA was 272 . The amount of time between consecutive urine samples ranged from 3 to 75 days, with a median $\left(25^{\text {th }}, 75^{\text {th }}\right.$ percentile $)$ of $29(27,34)$ days. Demographic variables stratified by semen quality reference values are presented in Table 1 . None of the demographic variables significantly differed between semen quality groups ( $\mathrm{p}$-values $>0.05$ ). The distribution of semen quality, sperm motion, and sperm DNA damage measures are presented in Table 2. Because the comet assay was introduced into the study at a later time than the assessment of semen quality, DNA damage measures were only available for 132 of the men. The distribution of urinary BPA concentrations for the 194 urine samples collected on the same day as the semen sample are presented in Table 3. BPA was detected in $89 \%$ of urine samples analyzed in the study. The geometric mean BPA concentration in the present study $(1.4 \mathrm{ng} / \mathrm{mL})$ was significantly lower than the geometric mean concentration from adult men in the same age range (18-55 years) from the US general population reported in NHANES 2005-06 ( $2.3 \mathrm{ng} / \mathrm{mL} ; \mathrm{p}<0.00001)$. Among the 78 men from whom 2 urine samples were collected, BPA concentrations in the two samples were weakly correlated (Spearman $r=$ 0.18 ; $\mathrm{p}$-value $=0.10$ ). Limiting this analysis to urine samples collected within the median duration between repeated urine sample collection within the same individual (29 days) did not significantly strengthen this correlation. 
When considering semen quality, sperm motion parameters, and DNA damage measures as continuous variables, BMI was inversely associated with Tail\% and abstinence period was positively associated with total sperm count and sperm concentration (p-values $<0.05$ ). Geometric mean uncorrected urinary BPA concentration was also higher among men whose urine sample was collected in the afternoon $(1.6 \mathrm{ng} / \mathrm{mL})$ compared to men providing the urine sample in the morning $(1.1 \mathrm{ng} / \mathrm{mL} ; \mathrm{p}=0.02)$. The difference in BPA concentrations between urine samples collected in the afternoon and morning was slightly lessened when comparing SG-corrected concentrations $(1.9 \mathrm{ng} / \mathrm{mL}$ vs. $1.5 \mathrm{ng} / \mathrm{mL} ; \mathrm{p}=0.09)$.

Crude logistic and linear regression results (not shown) were similar to the adjusted results presented in Tables 4 and 5. All logistic/linear regression results in Tables 4 and 5 were adjusted for urinary specific gravity, age, BMI, current smoking, abstinence period, and time of day of clinic visit (time of urine/semen sample collection). As shown in Table 4, there were no consistent relationships between urinary BPA concentrations and odds for below reference semen quality parameters, though odds ratios were somewhat elevated for below reference sperm concentration in statistical approach 1 (first column of odds ratios) and for below reference sperm motility and morphology in statistical approach 4 (fourth column of odds ratios). Results from statistical approach 3 (third column of odds ratios) differed as reduced odds were found, though the reported odds ratios were not statistically significant and considered unstable due to the reduced sample size.

In multivariable linear regression models using only urinary BPA concentrations measured in the urine sample collected on the same day as the semen sample (statistical approach 1, first column of regression coefficients in Table 5), we observed inverse associations between urinary BPA concentrations and sperm concentration, motility, morphology, VSL and VCL. For the median values of sperm concentration (64 million/mL), motility (49\% motile), and morphology (7\% normal) for the study population, the coefficients represent $23 \%$ (95\% CI $-40 \%$ to $-0.3 \%), 7.5 \%$ (95\% CI $-17 \%$ to $+1.5 \%$ ), and $13 \%$ (95\% CI $-26 \%$ to $-0.1 \%$ ) declines in these parameters, respectively, for an IQR increase in urinary BPA concentration (IQR 0.8 to $2.5 \mathrm{ng} / \mathrm{mL}$ ). Urinary BPA concentration was also positively associated with Tail\%, where an IQR increase in concentration was associated with a $10 \%$ (95\% CI $0.03 \%$ to $19 \%$ ) increase in Tail\% relative to the study population median. In sensitivity analyses, effect estimates from the multivariable models were similar when including only men with $\mathrm{SG} \geq$ 1.01 and $\leq 1.03$ were included ( $n=154$ for semen quality parameters, $n=105$ for DNA damage measures; results not shown). Results were also similar when modeling SGcorrected BPA concentrations as the independent variable as compared to including SG as a covariate when modeling uncorrected BPA concentrations (not shown).

When urinary BPA concentrations were categorized into quartiles to assess potential nonlinear relationships, there was no evidence for elevated odds ratios for below reference semen quality parameters in logistic regression models (not shown). Conversely, in multivariable linear regression models of semen quality parameters as continuous dependent variables, there were suggestive trends for non-monotonic declines in sperm concentration, motility and morphology, and a significant increasing trend in Tail\%, when categorizing urinary BPA concentration into quartiles (Figure $1 \mathrm{a}-\mathrm{d}$ ). BPA quartiles were not associated with total sperm count (not shown; $p$-value for trend $=0.57$ ). There were also statistically suggestive, though non-monotonic, relationships between urinary BPA concentration quartiles and reduced VSL, VCL, and LIN (results not shown; p-values for trend ranged from 0.05 to 0.13 ).

Results from the multivariable regression models using geometric mean BPA concentrations from multiple urine samples per participant (statistical approaches 2 and 3, the second and third columns of effect estimates in Tables 4 and 5), differed somewhat from the models 
using only urine samples collected on the same day as the serum samples. However, with the exception of the models for sperm concentration and motility using statistical approach 3 , in which effect estimates changed in sign or "direction", effect estimates were consistent though somewhat attenuated compared to statistical approach 1. Effect estimates obtained from approach 4 (using only BPA concentration from the same day as the semen sample and only from men with at least 2 urine samples) were also consistent with approaches 1,2 , and 3 , with the exception of sperm concentration and motility effect estimates from approach 3 . Wider confidence intervals were observed with approaches 3 and 4 as a result of the smaller sample size, and these effect estimates should be considered less stable.

Finally, we observed differences in effect estimates from multivariable linear regression models among (i.e., stratified by) men with $(n=114)$ or without $(n=76)$ at least one semen parameter below WHO reference values. When considering only BPA concentrations measured in urine samples collected on the same day as the semen sample (statistical approach 1), an IQR increase urinary BPA was associated with a 27\% decline in sperm concentration ( $p=0.048)$, a $6 \%$ decline in sperm motility $(p=0.2)$, and a $16 \%$ decline in sperm morphology $(\mathrm{p}=0.04)$ among men with at least one semen parameter below WHO reference values compared to little change $[-2 \%(p=0.8),-2 \%(p=0.6)$, and $+1 \%(p=0.9)$ for sperm concentration, motility and morphology, respectively], among men with all semen parameters above WHO reference values. These results were consistent when using geometric mean BPA concentrations (statistical approach 2), as the associations between urinary BPA and sperm concentration $(\mathrm{p}=0.06)$, motility $(\mathrm{p}=0.10)$, and morphology $(\mathrm{p}=$ 0.03 ) were stronger among men with at least one parameter below WHO reference compared to men with all parameters above WHO reference (all p-values > 0.4).

\section{DISCUSSION}

We found that urinary BPA concentrations measured in spot urine samples collected on the same day as a semen sample were associated with suggestive declines in semen quality parameters and with increased sperm DNA damage (measured as Tail\%). For the semen quality parameters, these associations were only observed when modeling the parameters as continuous variables in linear regression models but not when dichotomizing them according to widely accepted WHO reference values in logistic regression models. Also, in linear regression models stratified by semen quality status, the relationships were only found among men with at least one semen parameter below WHO reference values. When one or two subsequent urine samples collected in the weeks and months following the semen sample were taken into consideration the associations were inconsistent and weakened, perhaps due to reduced statistical power since repeated urines were only available among a subset of the men, or because the repeated samples were collected outside the most relevant exposure window of interest for these outcome measures (i.e. weeks and months prior to the semen sample).

Our findings of suggestive relationships between urinary BPA concentrations and semen quality parameters are consistent with animal studies reporting adverse effects on Sertoli cell function and sperm production in relation to BPA exposure [19-29]. Though some of these studies were conducted in vitro or report effects stemming from in utero or early postnatal BPA exposure, and thus limit our ability to directly compare with our findings, several studies have assessed effects following in vivo exposure in adulthood [20-23]. Sakaue et al. [20] showed that oral administration of BPA to adult Sprague-Dawley rats at concentrations as low as $20 \mu \mathrm{g} / \mathrm{kg}$ bw/day resulted in a reduction in daily sperm production of up to $40 \%$. This was confirmed in a later study on adult Swiss mice exposed to $25-100 \mathrm{ng} / \mathrm{kg}$ bw/day for one month. Daily sperm production, epididymal sperm concentration, and fertility were significantly decreased in the exposed groups compared to controls [21]. Toyama et al. [22] 
also noted spermatid abnormalities in adult Wistar rats and CD-1 mice injected with $20 \mu \mathrm{g} /$ $\mathrm{kg}$ bw/day for 6 days. Finally, there was a dose-dependent reduction in epididymal sperm motility and sperm count in male rats following ingestion of $0.2,2$, and $20 \mu \mathrm{g} / \mathrm{kg}$ bw/day of BPA for a period of 45 days [23].

In the present study, we found evidence for an association between urinary BPA concentrations and increased Tail\%, but no associations between urinary BPA concentrations and comet extent or TDM. Inconsistent results between the various DNA damage measures obtained by the neutral comet assay in relation to the same independent variable have been observed in previous studies, and it has been hypothesized that the different comet assay parameters may reflect different types of DNA strand breaks [30]. Specifically, a lack of correlation between TDM and Tail\% has been reported, and it was hypothesized that a high TDM may be more likely to be associated with double-strand breaks, whereas a high Tail\% may reflect single-strand breaks [30]. Thus, in the present study, the positive association between urinary BPA concentration and Tail\% may reflect a relationship between BPA exposure and single strand breaks. While BPA has expressed genotoxicity in a number of in vitro and in vivo models,31,32 findings have not been fully consistent and details of the direct mechanisms involved remain unclear. However, the adverse effects of BPA on adult male reproduction may be through the induction of oxidative stress and depletion of antioxidant defense mechanisms, as was reported in epididymal sperm of rats orally dosed with BPA [23].

Urinary BPA concentrations in the present study were lower than those reported for adult men in the same age range from the US general population in NHANES 2005-2006 (Table 3 ). In our study the median and $95^{\text {th }}$ percentile BPA concentrations (uncorrected for specific gravity) were $1.3 \mathrm{ng} / \mathrm{mL}$ and $9.3 \mathrm{ng} / \mathrm{mL}$, respectively, compared to $2.3 \mathrm{ng} / \mathrm{mL}$ and $12.2 \mathrm{ng} /$ $\mathrm{mL}$ among the 540 male participants aged 18-55 years in NHANES 2005-2006. One potential reason for the discrepancy may involve the inclusion of urine samples collected in the evening in NHANES, which had higher BPA concentrations than samples collected in the afternoon in earlier NHANES datasets [4]. In addition, differences in the distribution of race/ethnicity and income between the two study populations may also contribute to the difference in urinary BPA concentrations. For example, in NHANES 2003-04 urinary BPA concentrations were higher among non-hispanic blacks compared to non-hispanic whites, and higher among participants with lower household incomes [4]. Differences in study years should also be considered. Urine samples in the present study were collected in years 2000 to 2004 compared with 2005-06 in the most recent NHANES data. However, urinary BPA levels reported in the previous NHANES investigation (NHANES 2003-04) were even higher than in NHANES 2005-06 [4].

Since the present study was conducted among men recruited through an infertility clinic it may limit our ability to generalize the results to the general population. However, the men were members of couples seeking infertility diagnosis and treatment potentially related to a male factor, a female factor, or both, resulting in a study population that included both fertile men and men with a range of fertility problems. In addition, in order for the generalizability of our results to be limited, men recruited through an infertility clinic would have to respond differently (i.e. be more or less susceptible) to BPA exposure compared to men not in couples seeking infertility diagnosis/treatment. Although we are not aware of evidence showing that men from an infertility clinic are more sensitive to BPA exposure, we did find stronger relationships between urinary BPA concentrations and reduced semen quality parameters among men with at least one parameter below WHO reference values. Thus, our data suggest the possibility that men with subfertility are more sensitive to BPA-related effects than men with normal fertility. As more studies are published on BPA exposure and 
semen quality, it is important to consider the subject populations when interpreting the results.

We found that repeated urine samples collected from the same man weeks to months apart from one another were weakly correlated $(\mathrm{r}=0.18)$. Thus, another limitation in the present study is the likelihood of exposure measurement error due to the high within-individual temporal variability in BPA exposure and the availability of multiple BPA measures from only a subset of participants. However, measurement error would be expected to be nondifferential, which would tend to reduce the ability to detect associations between exposure and outcome. In addition, when using broad exposure categories (e.g. quartiles in Figure 1), a single measure may adequately predict an individual's exposure category over a longer period of time [7]. We also collected only a single semen sample from each man, and the reliability of a single semen sample to represent semen quality over a longer period of time is not well characterized. However, two recent reports provide evidence that one sample may be representative of semen quality over several weeks in large epidemiologic studies $[33,34]$.

Another limitation of our study was its cross-sectional design due to the availability of only a single semen sample from each participant, as well as the availability of only a single urine sample from over half of the men. Thus, we cannot rule out reverse causation in the explanation of our findings in the event that an underlying condition that causes poor semen quality may also lead to altered BPA metabolism.

In conclusion, human exposure to BPA may be associated with reduced semen quality and increased sperm DNA damage. However, due to some inconsistencies in our results between statistical and exposure assessment approaches, these relationships need to be assessed in other large and appropriately designed human epidemiologic studies that measure BPA in multiple urine samples across the exposure window of interest.

\section{Acknowledgments}

Work supported by grants ES009718 and ES00002 from the National Institute of Environmental Health Sciences (NIEHS), National Institutes of Health (NIH). The authors gratefully acknowledge Amber Bishop, Tao Jia, and Jack Reidy (CDC, Atlanta, GA) for measuring the urinary concentrations of BPA. Disclaimer: The findings and conclusions in this report are those of the author and do not necessarily represent the official position of the Centers for Disease Control and Prevention.

\section{REFERENCES}

1. Vandenberg LN, Hauser R, Marcus M, Olea N, Welshons WV. Human exposure to bisphenol A (BPA). Reprod Toxicol 2007;24(2):139-77. [PubMed: 17825522]

2. Burridge E, Bisphenol A. product profile. Eur Chem News 2003;17:14-20.

3. Geens T, Roosens L, Neels H, Covaci A. Assessment of human exposure to Bisphenol A, Triclosan and Tetrabromobisphenol-A through indoor dust intake in Belgium. Chemosphere 2009;76(6):75560. [PubMed: 19535125]

4. Calafat AM, Ye X, Wong LY, Reidy JA, Needham LL. Exposure of the U.S. population to bisphenol A and 4-tertiary-octylphenol: 2003-2004. Environ Health Perspect 2008;116(1):39-44. [PubMed: 18197297]

5. Wetherill YB, Akingbemi BT, Kanno J, McLachlan JA, Nadal A, Sonnenscein C, et al. In vitro molecular mechanisms of bisphenol A action. Reprod Toxicol 2007;24(2):178-98. [PubMed: 17628395]

6. Richter CA, Birnbaum LS, Farabollini F, Newbold RR, Rubin BS, Talsness CE, et al. In vivo effects of bisphenol A in laboratory rodent studies. Reprod Toxicol 2007;24(2):199-224. [PubMed: 17683900] 
7. Mahalingaiah S, Meeker JD, Pearson KR, Calafat AM, Ye X, Petrozza J, et al. Temporal variability and predictors of urinary bisphenol A concentrations in men and women. Environ Health Perspect 2008;116(2):173-8. [PubMed: 18288314]

8. Ye X, Kuklenyik Z, Needham LL, Calafat AM. Automated on-line column-switching HPLC-MS/ MS mkethod with peak focusing for the determination of nine environmental phenols in urine. Anal Chem 2005;77(16):5407-13. [PubMed: 16097788]

9. Duty SM, Singh NP, Ryan L, Chen Z, Lewis C, Huang T, et al. Reliability of the comet assay in cryopreserved human sperm. Hum Reprod 2002;17(5):1274-80. [PubMed: 11980751]

10. World Health Organization (WHO). WHO Laboratory Manual for the Examination of Human Semen and Sperm-Cervical Mucus Interaction. Cambridge University Press; New York: 1999.

11. Meeker JD, Ryan L, Barr DB, Herrick RF, Bennett DH, Bravo R, et al. The relationship of urinary metabolites of carbaryl/naphthalene and chlorpyrifos with human semen quality. Environ Health Perspect 2004;112(17):1665-70. [PubMed: 15579410]

12. Duty SM, Calafat AM, Silva MJ, Brock JW, Ryan L, Chen Z, et al. The relationship between human environmental exposure to phthalates and computer aided sperm analysis motion parameters. J Androl 2004;25(2):293-302. [PubMed: 14760016]

13. Kruger TF, Acosta AA, Simmons KF, Swanson RJ, Matta JF, Oehninger S. Predictive value of abnormal sperm morphology in in vitro fertilization. Fertil Steril 1988;49(1):112-7. [PubMed: 3335257]

14. Singh NP, Stephens RE. X-ray-induced DNA double-strand breaks in human sperm. Mutagenesis 1998;13(1):75-9. [PubMed: 9491398]

15. Duty SM, Singh NP, Silva MJ, Barr DB, Brock JW, Ryan L, et al. The relationship between environmental exposures to phthalates and DNA damage in human sperm using the neutral comet assay. Environ Health Perspect 2003;111(9):1164-9. [PubMed: 12842768]

16. National Health and Nutrition Examination Survey. (NHANES) 2005-06 [Internet]. CDC; Atlanta (GA): [2009 Sept 23]. c2009 -. Available from: http://www.cdc.gov/nchs/nhanes/nhanes2005-2006/nhanes05_06.htm

17. Kleinbaum, DG.; Kupper, LL.; Muller, KE.; Nizam, A. Applied regression analysis and other multivariate methods. 3rd ed.. Brooks/Cole Publishing Company; Pacific Grove (CA): 1998. Selecting the best regression equation..

18. Teass, AW.; Biagini, RE.; DeBord, DG.; Hull, RD. NIOSH Manual of Analytical Methods. National Institute for Occupational Safety and Health; Cincinnati: 1998. Application of biological monitoring methods.; p. 52-62.

19. Hughes PJ, McLellan H, Lowes DA, Kahn SZ, Bilmen JG, Tovey SC, et al. Estrogenic alkylphenols induce cell death by inhibiting testis endoplasmic reticulum $\mathrm{Ca}(2+)$ pumps. Biochem Biophys Res Commun 2000;277(3):568-74. [PubMed: 11061995]

20. Sakaue M, Ohsako S, Ishimura R, Kurosawa S, Kurohmaru M, Hayashi Y, et al. Bisphenol A affects spermatogenesis in the adult rat even at a low dose. J Occup Health 2001;43:185-90.

21. Al-Hiyasat AS, Darmani H, Elbetieha AM. Effects of bisphenol A on adult male mouse fertility. Eur J Oral Sci 2002;110(2):163-7. [PubMed: 12013561]

22. Toyama Y, Suzuki-Toyota F, Maekawa M, Ito C, Toshimori K. Adverse effects of bisphenol A to spermiogenesis in mice and rats. Arch Histol Cytol 2004;67(4):373-81. [PubMed: 15700544]

23. Chitra KC, Latchoumycandane C, Mathur PP. Induction of oxidative stress by bisphenol A in the epididymal sperm of rats. Toxicology 2003;185(1-2):119-27. [PubMed: 12505450]

24. Tabuchi Y, Zhao QL, Kondo T. DNA microarray analysis of differentially expressed genes responsive to bisphenol A, an alkylphenol derivative, in an in vitro mouse Sertoli cell model. Jpn J Pharmacol 2002;89(4):413-6. [PubMed: 12233820]

25. Tabuchi Y, Kondo T. cDNA microarray analysis reveals chop-10 plays a key role in Sertoli cell injury induced by bisphenol A. Biochem Biophys Res Commun 2003;305(1):54-61. [PubMed: 12732195]

26. Tabuchi Y, Takasaki I, Kondo T. Identification of genetic networks involved in the cell injury accompanying endoplasmic reticulum stress induced by bisphenol A in testicular Sertoli cells. Biochem Biophys Res Commun 2006;345(3):1044-50. [PubMed: 16713995] 
27. Aikawa H, Koyama S, Matsuda M, Nakahashi K, Akazome Y, Mori T. Relief effect of vitamin A on the decreased motility of sperm and the increased incidence of malformed sperm in mice exposed neonatally to bisphenol A. Cell Tissue Res 2004;315(1):119-24. [PubMed: 14557871]

28. Akingbemi BT, Sottas CM, Koulova AI, Klinefelter GR, Hardy MP. Inhibition of testicular steroidogenesis by the xenoestrogen bisphenol $\mathrm{A}$ is associated with reduced pituitary luteinizing hormone secretion and decreased steroidogenic enzyme gene expression in rat Leydig cells. Endocrinology 2004;145(2):592-603. [PubMed: 14605012]

29. Toyama Y, Yuasa S. Effects of neonatal administration of 17beta-estradiol, beta-estradiol 3benzoate, or bisphenol A on mouse and rat spermatogenesis. Reprod Toxicol 2004;19(2):181-8. [PubMed: 15501383]

30. Meeker JD, Singh NP, Ryan L, Duty SM, Barr DB, Herrick RF, et al. Urinary levels of insecticide metabolites and DNA damage in human sperm. Hum Reprod 2004;19(11):2573-80. [PubMed: 15333606]

31. Chapin RE, Adams J, Boekelheide K, Gray LE Jr. Hayward SW, Lees PS, et al. NTP-CERHR expert panel report on the reproductive and developmental toxicity of bisphenol A. Birth Defects Res B Dev Reprod Toxicol 2008;83(3):157-395. [PubMed: 18613034]

32. Naik P, Vijayalaxmi KK. Cytogenetic evaluation for genotoxicity of bisphenol-A in bone marrow cells of Swiss albino mice. Mutat Res 2009;676(1-2):106-12. [PubMed: 19393337]

33. Francavilla F, Barbonetti A, Necozione S, Santucci R, Cordeschi G, Macerola B, et al. Withinsubject variation of seminal parameters in men with infertile marriages. Int J Androl 2007;30(3): 174-81. [PubMed: 17239086]

34. Stokes-Riner A, Thurston SW, Brazil C, Guzick D, Liu F, Overstreet JW, et al. One semen sample or 2? Insights from a study of fertile men. J Androl 2007;28(5):638-43. [PubMed: 17522415] 


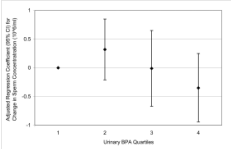

b.
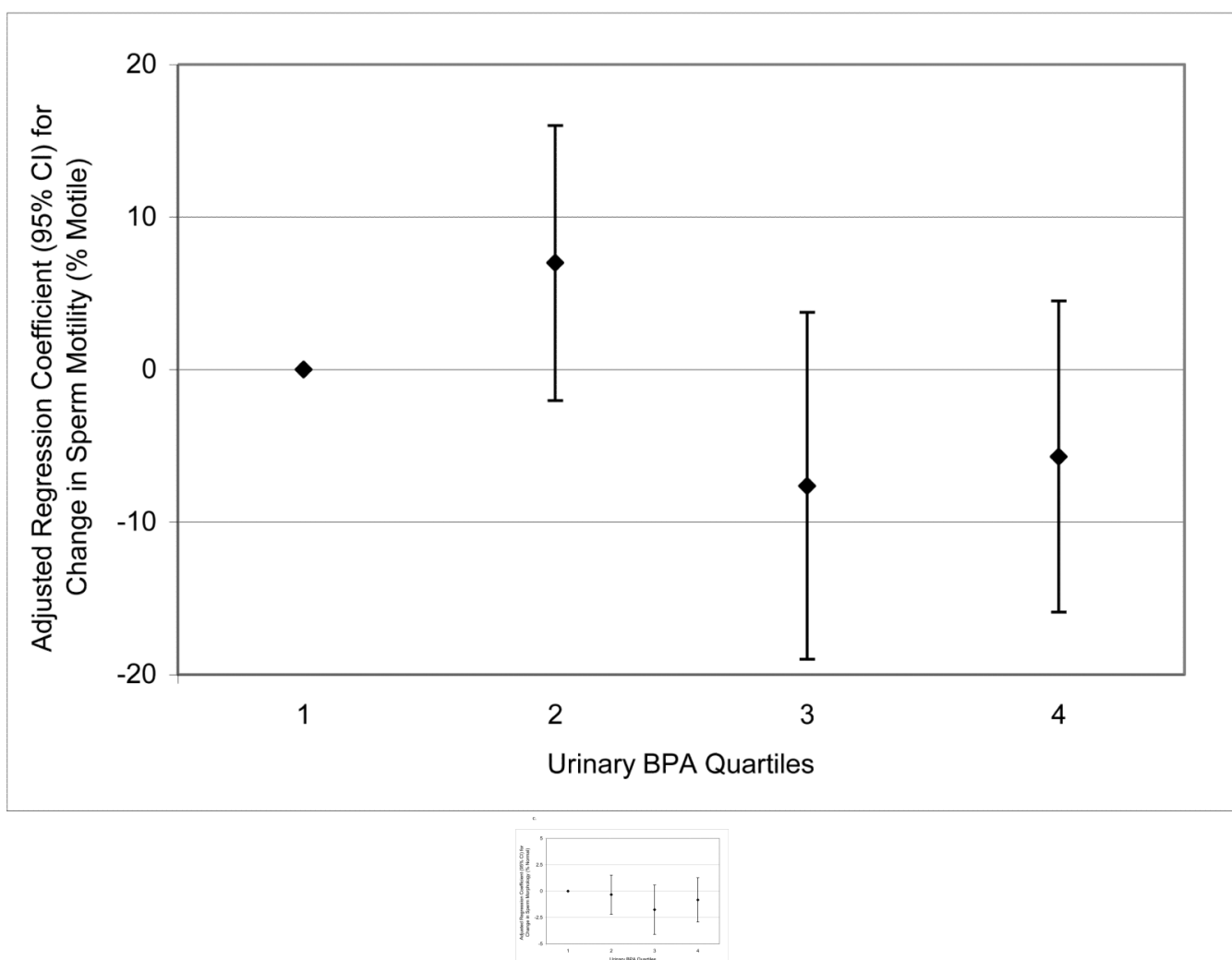

Figure 1.

Adjusted regression coefficients for a change in semen quality parameter or sperm DNA damage measure associated with increasing quartiles of urinary BPA concentration $(n=190)$ : a) sperm concentration ( $\mathrm{p}$-value for trend $=0.09$ ); ) sperm motility ( $\mathrm{p}$-value for trend $=$ $0.04)$; c) sperm morphology ( $\mathrm{p}$-value for trend $=0.13$ ); ) Tail\% ( $\mathrm{p}$-value for trend $=0.03$ ). Adjusted for specific gravity, age, BMI, abstinence period, current smoking status, and time of day of urine sample. 


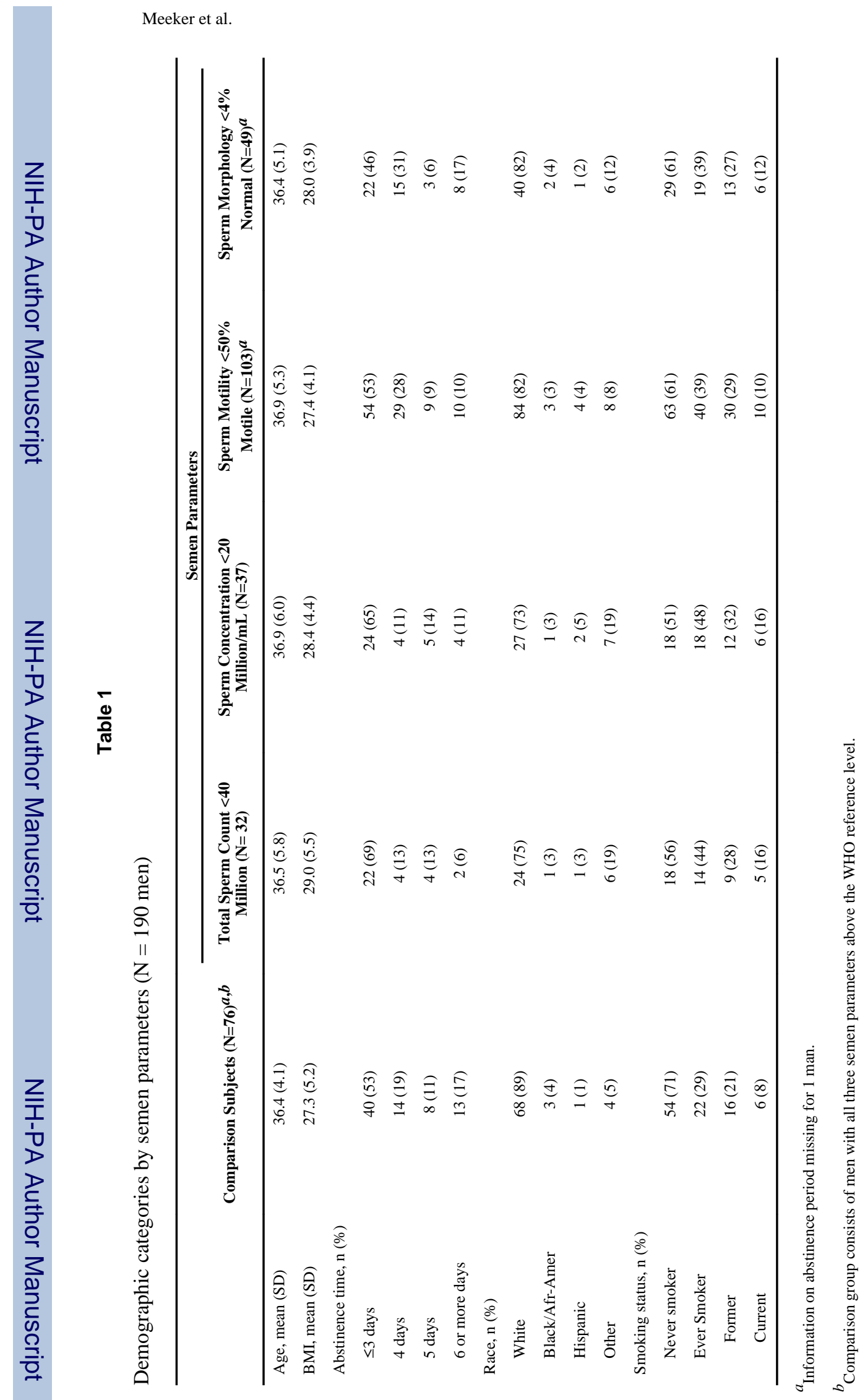

Page 13 


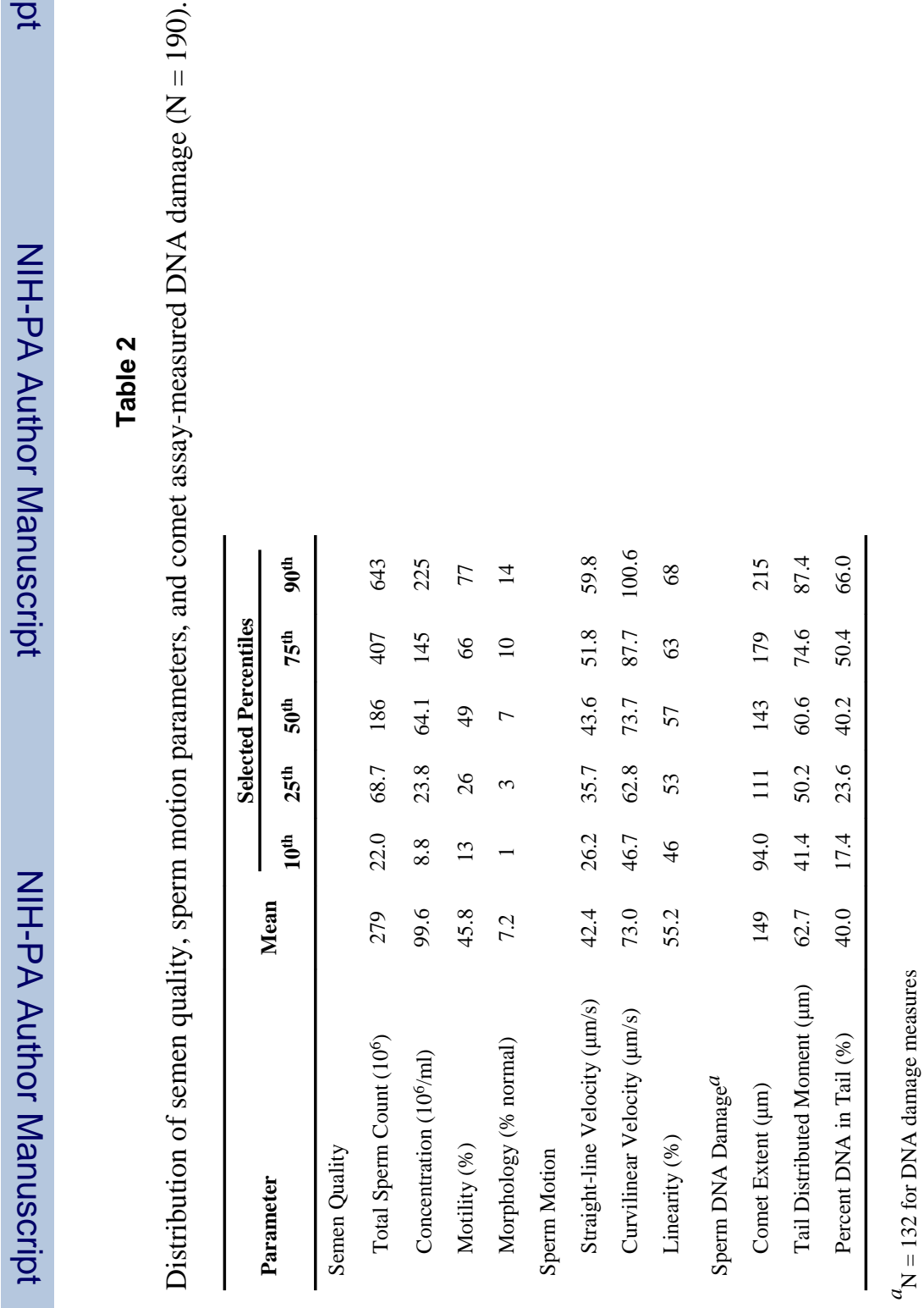

Reprod Toxicol. Author manuscript; available in PMC 2011 December 1. 


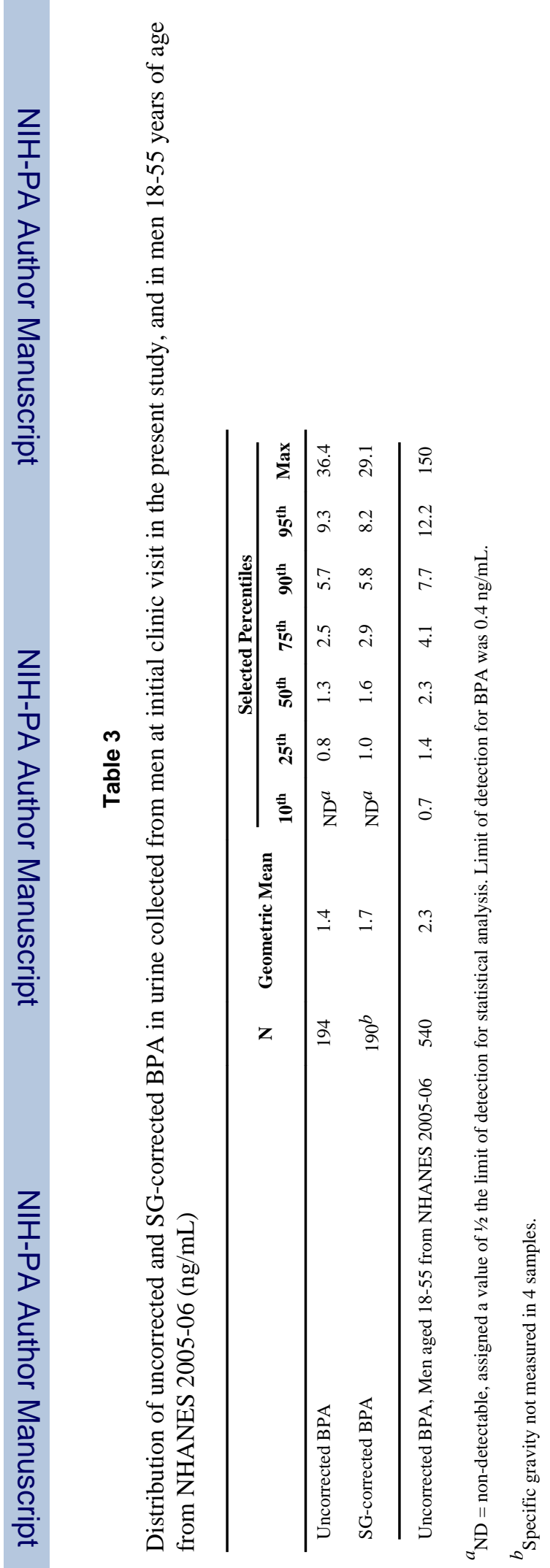

Reprod Toxicol. Author manuscript; available in PMC 2011 December 1. 


\section{Table 4}

Adjusted odds ratios (95\% confidence intervals) for below reference semen quality parameter associated with an interquartile range increase in urinary BPA concentration. Adjusted for specific gravity, age, BMI, abstinence period, current smoking, and time of urine sample.

\begin{tabular}{|c|c|c|c|c|}
\hline Semen Quality Parameter & $\begin{array}{l}\text { 1) BPA measure from } \\
\text { same day as semen } \\
\text { sample only }(\mathrm{n}=190)^{a, d}\end{array}$ & $\begin{array}{l}\text { 2) Geometric Mean } \\
\text { BPA from all } \\
\text { participants } \\
(\mathrm{n}=190)^{b, d}\end{array}$ & $\begin{array}{l}\text { 3) Geometric mean } \\
\text { BPA from men with } \geq 2 \\
\text { BPA measures } \\
(\mathrm{n}=78)^{c, d}\end{array}$ & $\begin{array}{l}\text { 4) BPA from same day } \\
\text { as semen among men } \\
\text { with } \geq 2 \text { BPA measures } \\
(n=78)^{a, d}\end{array}$ \\
\hline & Odds Ratio (95\% CI) & Odds Ratio (95\% CI) & Odds Ratio (95\% CI) & Odds Ratio (95\% CI) \\
\hline Total Sperm Count $\left(<40 \times 10^{6}\right)$ & $1.20(0.71,2.03)$ & $0.98(0.57,1.68)$ & $0.30(0.04,2.69)$ & $1.50(0.36,6.22)$ \\
\hline $\begin{array}{l}\text { Sperm Concentration }(<20 \\
\text { million } / \mathrm{mL})\end{array}$ & $1.47(0.85,2.54)$ & $1.21(0.69,2.11)$ & $0.37(0.05,2.63)$ & $1.18(0.35,3.94)$ \\
\hline $\begin{array}{l}\text { Sperm Motility ( }<50 \% \text { motile } \\
\text { sperm) }\end{array}$ & $1.23(0.83,1.80)$ & $0.96(0.63,1.46)$ & $0.74(0.33,1.64)$ & $1.54(0.83,2.84)$ \\
\hline $\begin{array}{l}\text { Sperm Morphology }(<4 \% \\
\text { normal) }\end{array}$ & $1.25(0.77,2.06)$ & $1.01(0.60,1.72)$ & $0.98(0.35,2.70)$ & $1.70(0.73,3.95)$ \\
\hline
\end{tabular}

${ }^{a}$ Among men with urine sample from same visit as blood (hormone) sample only. $\mathrm{N}=190$.

${ }^{b}$ Geometric mean of up to 3 repeated urine samples per subject, some of which were collected months after semen sample. N=190.

${ }^{c}$ Geometric mean value among men with at least 2 urine samples. $\mathrm{N}=78$.

$d_{\text {Ln-transformations of bisphenol A concentration were used in the models. }}$ 
Meeker et al.

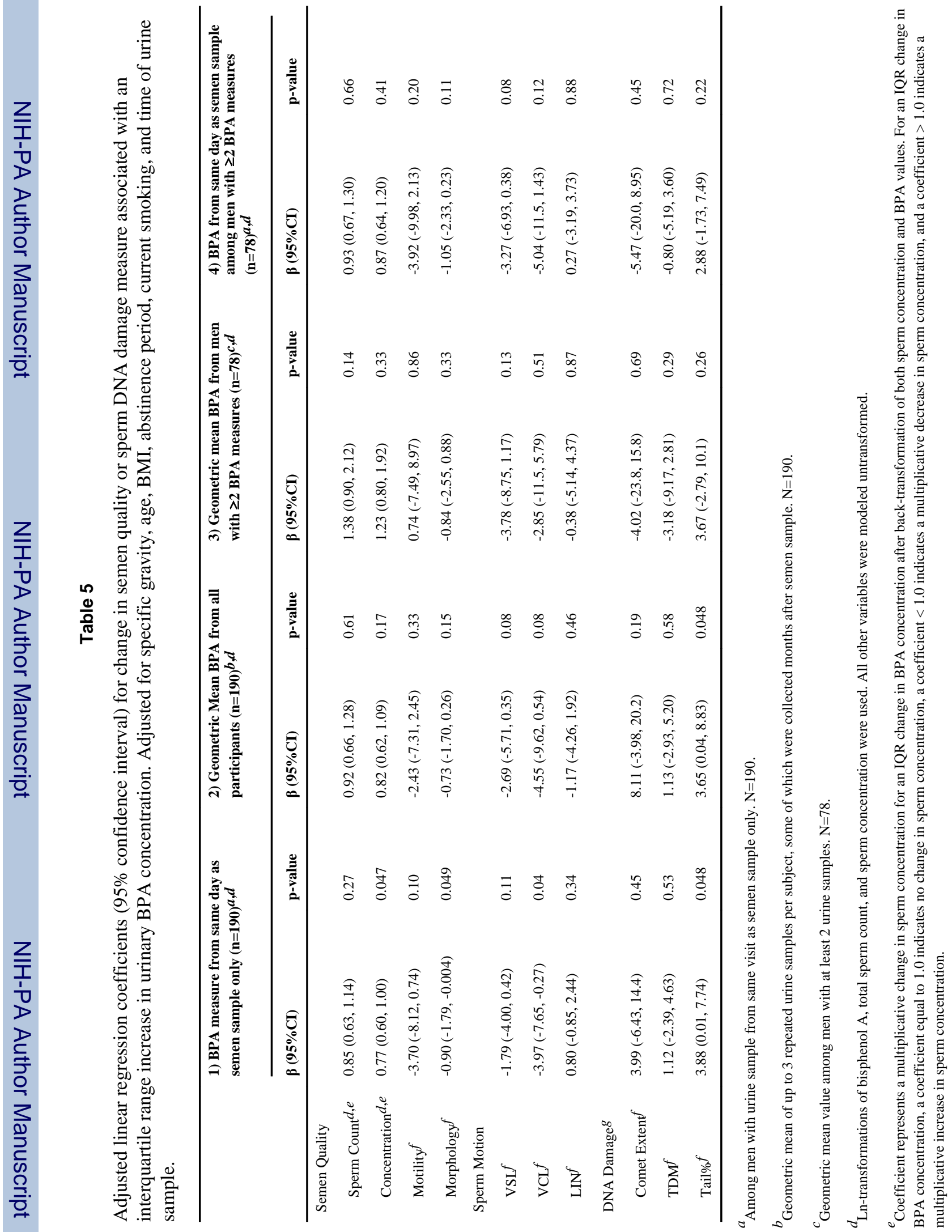




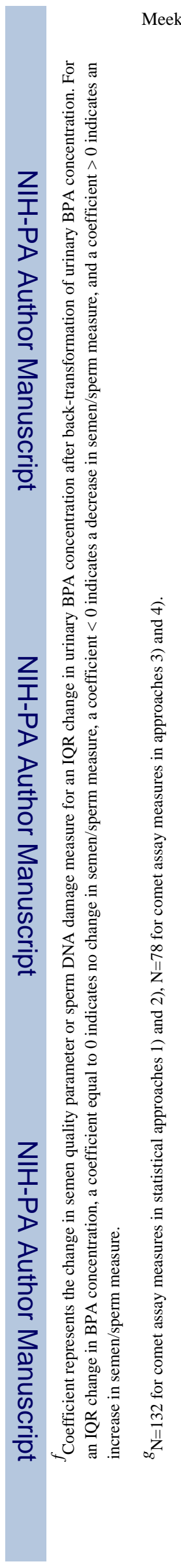

Page 18

Reprod Toxicol. Author manuscript; available in PMC 2011 December 1. 